\title{
Experiences of thoracic esophagectomy with laparoscopic gastric pull up in thoracic esophageal cancer patient in single center
}

\author{
Jin-Woo Jun', Wooshik Kim², Jong-Min Park' \\ Departments of ${ }^{1}$ Surgery and ${ }^{2}$ Thoracic \& Cardiovascular Surgery, National Medical Center, Seoul, Korea
}

Purpose: The standard treatment of esophageal cancer is the Ivor-Lewis operation, which consists of an abdominal phase involving gastric tube formation, and a chest phase involving esophagectomy and anastomosis. We aimed to report our experience of performing thoracic esophagectomy with the laparoscopic gastric pull up (LGPU) technique and its surgical outcomes.

Methods: Clinicopathologic data and short-term surgical outcomes of 14 patients who underwent LGPU for thoracic esophageal cancer from August 2008 to May 2016 were retrospectively reviewed.

Results: Mean age of the patients was 62.3 years and mean body mass index was $21.7 \mathrm{~kg} / \mathrm{m}^{2}$. Eleven patients had medical comorbidities. Patients' mean American Society of Anesthesiologists score was 2. Mean operation time was 428.5 minutes, with the mean abdominal operation time being 138.9 minutes. There was no open conversion case. Three patients had pneumonia, three patients had surgical site infection, and one patient had subcutaneous emphysema within 30 days after surgery. One patient had minor anastomosis site leakage. There was one 30-day mortality case. One patient with postoperative aspiration pneumonia developed acute respiratory distress disease, and died due to sepsis. Mean postoperative intensive care unit stay was 3.5 days, and mean postoperative hospital stay was 20.6 days. Nasogastric tubes were removed on average at 3.4 days, and mean oral intake time was 3.4 days.

Conclusion: If the gastrointestinal surgeon has extensive experience in laparoscopic procedures, LGPU will be a safe and feasible technique for thoracic esophagectomy in patients with intrathoracic esophageal cancer.

Keywords: Esophageal neoplasm, Laparoscope, Esophagectomies, Reconstructive surgical procedures

\section{INTRODUCTION}

Metastasis of esophageal cancer to adjacent organs commonly occurs due to the esophagus' anatomical characteristic, wherein the serosa is absent, thereby making the surgical treatment difficult at the time of discovery. Nevertheless, when operable esophageal can-

Received: Aug 24, 2018 Revised: Oct 18, 2018 Accepted: Nov 08, 2018 Correspondence to: Jong-Min Park

Department of Surgery, National Medical Center, 245 Eulji-ro, Jung-gu, Seoul 04564, Korea

Tel: +82-2-2260-4883, Fax: +82-2-2269-0750

E-mail: jmparkgs@gmail.com

ORCID: Jin-Woo Jun (https://orcid.org/0000-0002-0169-9744), Wooshik Kim (https://orcid.org/0000-0003-2787-2186), Jong-Min Park (https://orcid.org/00000003-4023-5560)

Copyright (C) 2018 Korean Society of Surgical Oncology

This is an Open Access article distributed under the terms of the Creative Commons Attribution Non-Commercial License (http://creativecommons.org/licenses/by-nc/4.0) which permits unrestricted non-commercial use, distribution, and reproduction in any medium, provided the original work is properly cited. cer is diagnosed, the gold standard for treatment is the Ivor-Lewis operation, which consists of esophagectomy, esophageal reconstruction, and anastomosis. The ideal conduit used for esophageal reconstruction should be an organ, requiring a relatively simple surgical technique, with similar size and function of the original esophagus, and with minimal complications after surgery [1]. The stomach has advantages over other organs like the colon and jejunum, such as appropriate length, lesser deviation in blood flow supply, easy to operate, and only requires a single anastomosis $[2,3]$.

Since the introduction of minimally invasive esophagectomy in the 1990s, video-assisted thoracic surgery has been widely used [4], though our center currently uses open esophagectomy and laparoscopic gastric pull up (LGPU). Laparoscopic surgery is widely used owing to its variety of benefits, such as reduced hospital stay, reduced postoperative pain, and better cosmetic aspect, since laparoscopic cholecystectomy surgery was introduced [5]. Our center utilized the open gastric pull-up method after esophagectomy in esophageal cancer treatment until May 2008. Conventional open 
surgery has several disadvantages, as compared to laparoscopic surgery as mentioned above, such as postoperative pain that makes respiration difficult. For these reasons, since August 2008, we have been performing the LGPU method.

In this study, we aimed to introduce our experiences in performing thoracic esophagectomy with LGPU in patients with intrathoracic esophageal cancer in a single center.

\section{METHODS}

\section{Patients}

From August 2008 to May 2016, patients' data were collected retrospectively using the electronic medical record (EMR) system for 14 esophageal cancer patients who underwent LGPU after esophagectomy at our center. Preoperative staging of all patients was based on endoscopic biopsy and abdominal and chest computed tomography. Disease stage was classified according to the 7th Union for International Cancer Control (UICC) TNM classification.

The surgical procedure was performed as follows. First, a gastrointestinal (GI) surgeon performed laparoscopic gastric mobilization and lymph node dissection, then the esophageal hiatus was peeled off, and the stomach was prepared to be pulled up. Subsequently, a thoracic surgeon performed open thoracotomy followed by intrathoracic lymph node dissection and esophageal mobilization. After the GI surgeon created a tube using the stomach pulled up via the esophageal hiatus, the operation was completed after esophago-gastric anastomosis was performed by the thoracic surgeon.

We retrospectively collected the patients' clinicopathologic outcomes, operation time, open conversion, blood loss, transfusion, postoperative complication and its Clavien-Dindo class, 30-day mortality, intensive care unit (ICU) stay, hospital stay, chest tube and nasogastric (NG) tube removal day, and diet start day. Accurate measurement of blood loss by EMR was not appropriate since the recorded volume was a combined record of washing fluid and blood. Comparison of the amount of blood loss recorded during surgery is a combined record of sap for washing and blood; thus, it was difficult to accurately measure the amount of blood loss. Hence, data on transfused packed red blood cell volume on the day after surgery was also collected.

All patients were informed about the procedure and consents for surgery were obtained. This study was approved by the Institutional Review Board of the National Medical Center (IRB No. H-1711-084-001).

\section{Abdominal phase: total laparoscopic}

All patients were intubated with a left-sided double-lumen tube to enable selective desufflation of the right lung during the thoracic phase in the procedure. The patient was placed in the supine position, while the operator and camera assistant stood on the right side of the patient and the 1st assistant stood on the patient's left side. After general anesthesia, a 10-mm trocar was inserted at the sub-umbilical area, and pneumoperitoneum was formed by insufflation of carbon dioxide. The intraperitoneal pressure was maintained at 12 to 15 $\mathrm{mmHg}$. Four additional trocars were inserted by direct visualization. One 5-mm port was inserted at the right upper quadrant (RUQ) and the other 5 -mm port was inserted at the left lower quadrant. One 10$\mathrm{mm}$ port was inserted at the right lower quadrant and the other 10$\mathrm{mm}$ port was inserted at the left upper quadrant (LUQ) (Fig. 1). The liver was retracted upward by a fan retractor via the LUQ port. The EXERA laparo-thoraco videoscope (Olympus, Tokyo, Japan) was used. For dissection of tissues, an ultrasonic cautery Harmonic scalpel (Ethicon Endo-Surgery Inc., Cincinnati, OH, USA) was used.

First, intraperitoneal laparoscopic exploration was performed. Afterwards, the patient was turned to the reverse Trendelenburg position. The gastroepiploic arcade was dissected by a harmonic scalpel, and omentectomy was performed (Fig. 2). The left gastroepiploic artery and short gastric artery were dissected by harmonic scalpel consecutively. The gastrocolic omentum was divided while preserving the right gastroepiploic artery. A generous kocherization was carried out all the way to the lower end of the duodenal loop; such peritoneal deflection allowed the whole distal stomach and duodenum to be pulled cephalad without tension after the right gastric artery dissection (Fig. 3). LGA was divided from its celiac trunk origin (Fig. 4). Then, the left crus of the diaphragm was dissected. The esophageal hiatus was dissected by harmonic scalpel after mobilization of the esophagus under the diaphragm (Fig. 5).

As it is well known, upper abdomen lymph node metastasis is

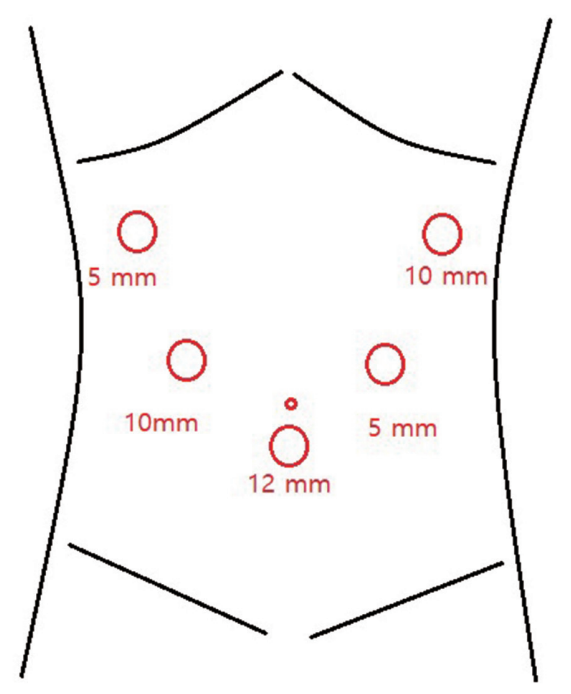

Fig. 1. Trocar placement on abdomen. 


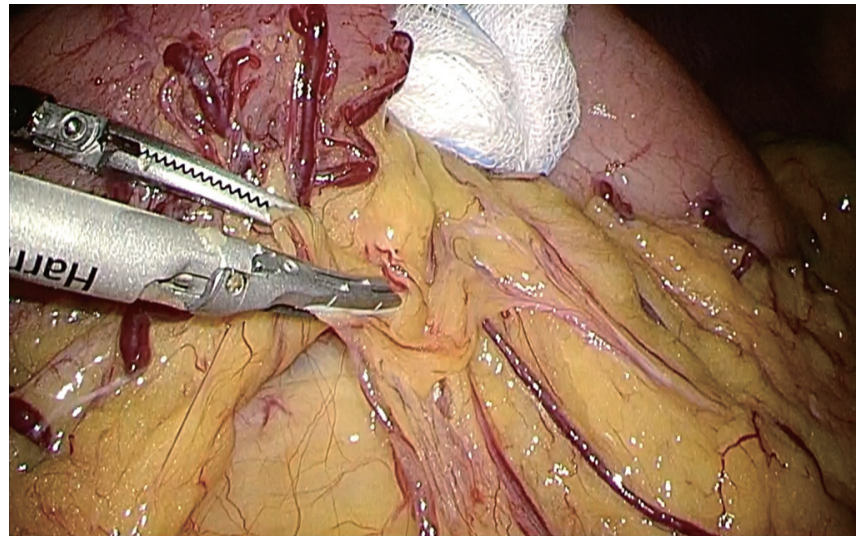

Fig. 2. Omentectomy. It shows the dissection and preservation of gastroepiploic arcade.

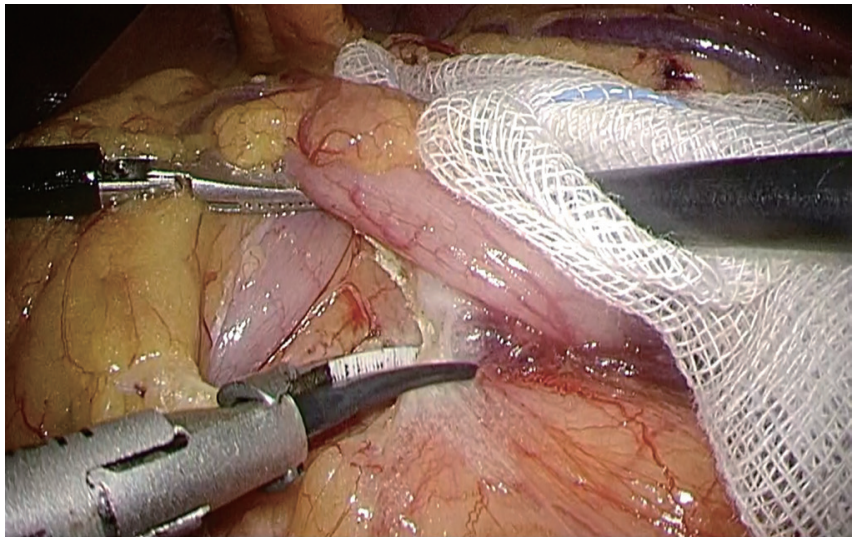

Fig. 3. Kocherization. It shows the kocherization of duodenal bulb.

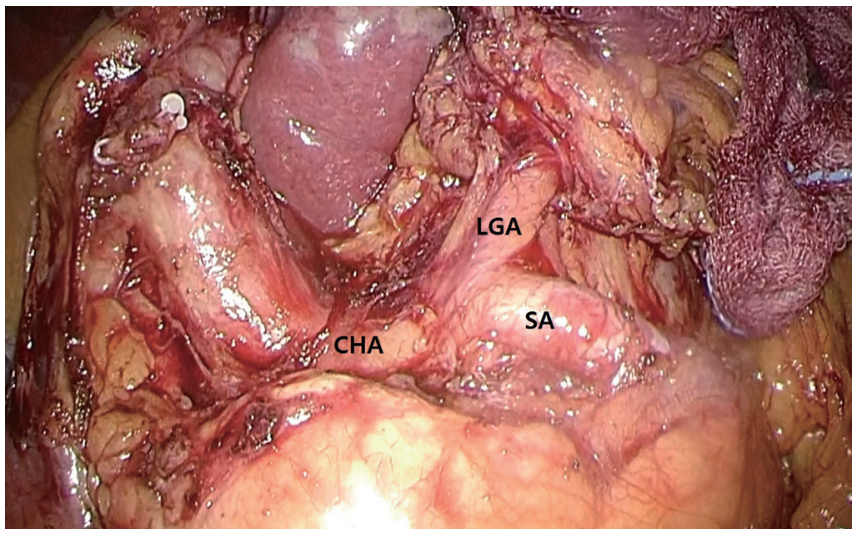

Fig. 4. Vessels. It is the laparoscopic view after lymph node dissection of the celiac trunk. CHA, common hepatic artery; LGA, left gastric artery; $\mathrm{SA}$, splenic artery.

frequently observed in thoracic esophageal cancer patients [6], the number 1, 2, 3, 4, and 7 lymph nodes were dissected according to the proximal gastrectomy D1 criteria including enlarged lymph node under visualization (Fig. 6). Pyloroplasty or pyloromyotomy

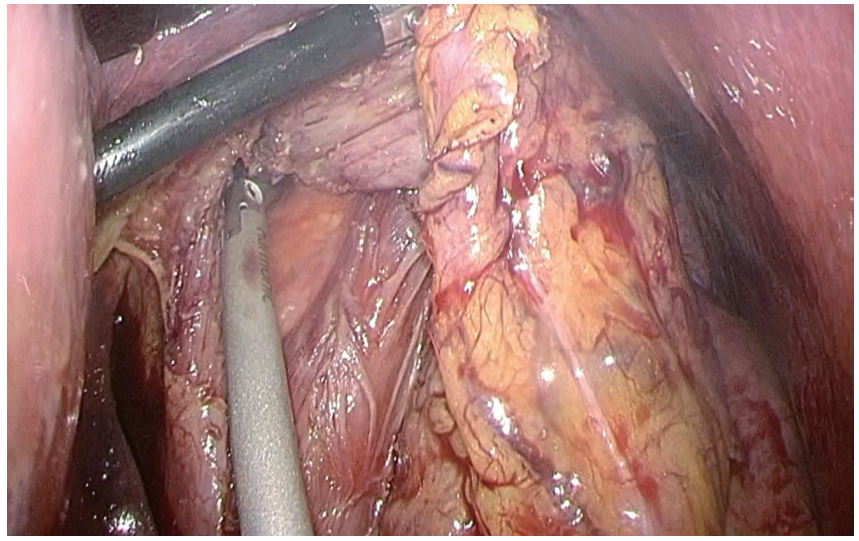

Fig. 5. Hiatus dissection. It shows dissection process of diaphragmatic hiatus.

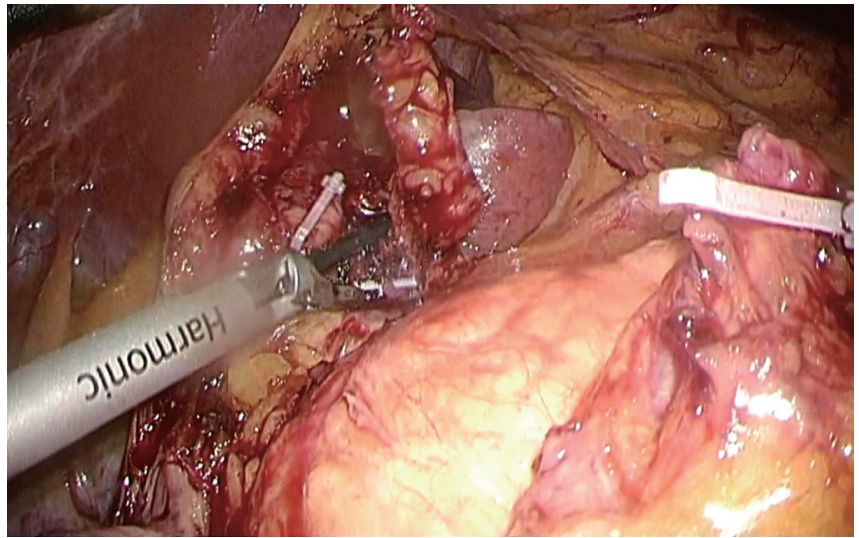

Fig. 6. Celiac lymph node dissection.

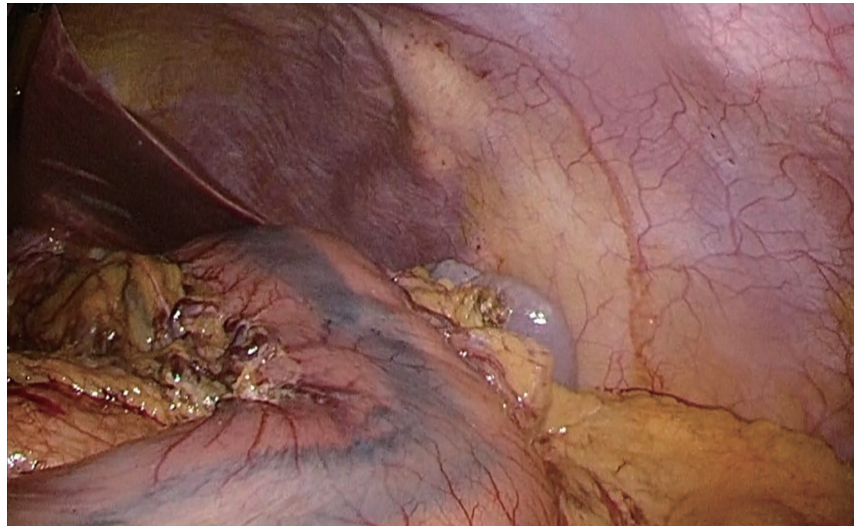

Fig. 7. Indigo-carmine painting on the stomach surface.

was not performed owing to its postoperative risk of perforation of up to $5.6 \%$ [7]; Despite of absence of pyloroplasty or pyloromyotomy, no patient had complained of postoperative dysphagia in our center. Linear painting on the stomach body using an indigo carmine solution to mark the margin of the stomach tube formation was done (Fig. 7). Thorough saline irrigation was performed, and 
one Jackson-Pratt drain was inserted via the 5-mm RUQ port site. Finally, trocar wounds were closed layer by layer.

\section{Thoracic and anastomosis phases}

Patients' were turned to the left lateral decubitus position. The position of the double-lumen tube was verified, and single-lung ventilation was used. The operator stood on the right side of the patient and the 1st assistant stood on the left side. Right posterolateral thoracotomy incision was performed, entering the chest through the 5th intercostal space. To secure visibility, the right 6 th rib was removed, and space was secured using a rib retractor. The right lung was collapsed through a one-lung ventilation technique. The azygos vein was stapled with a vascular stapler, Echelon Flex 35 $\mathrm{mm}$ (Ethicon, Bridgewater, NJ, USA), at the level of the azygos arch. The thoracic duct was identified, clipped, and ligated to prevent chylous leakage. The mediastinal pleura overlying the esophagus was opened via a bovie electrocauterization unit. Regional lymph nodes and soft tissue including the subcarinal and recurrent lymph nodes from the thoracic inlet to the diaphragm were dissected during the exfoliation of the esophagus. The thoracic duct was clipped at the lowest level as possible. The esophagus was clamped at the thoracic inlet level after the entire thoracic esophagus was mobilized. The clamped esophagus was transected by bovie. Purse string suture was done using prolene at the distal end of the esophagus, and then an EEA (Ethicon) stapler anvil (25 mm) was introduced into the esophagus. The purse string suture was secured.

The stomach was brought up through the esophageal hiatus into the right thoracic cavity. Linear stapling using three or four $45-\mathrm{mm}$ Endo GIA staplers (Tri-Stapler, Covidien, MN, USA) along the lesser curvature of the stomach was performed, and then the esophageal tumor and the stomach cardia were resected. A 25-mm circular stapler was placed transthoracically into the gastric tube, and construction of a stapled esophago-gastric anastomosis was performed. Careful squeezing of anvil and body was performed. Firm and sustained squeezing provided good intrathoracic anastomosis. We performed reinforcement suture at the anastomosis site of the esophagus and the stomach by using black silk 3-0. The stomach tube was fixed at the posterior pleura by using black silk 3-0. Then, two 32-Fr chest tubes were placed and the removed 6th rib was fixed at the original position. The thoracotomy wound was closed using intracutaneous closure with absorbable sutures. NG tube was routinely placed.

\section{Postoperative care}

Routine extubation in the operating room was attempted in all patients. We performed esophagography routinely at postoperative day 3. NG tube was removed if no leakage was found on the esophagogram. Clear fluid in small portions was started by mouth on about the 3rd postoperative day, if the abdomen was soft and peristalsis was present. Diet was changed gradually from the sips of water to a liquid diet and soft diet, when the patient did not have any GI problems. The patient routinely spent the first night in the ICU and was then transferred to the general ward. Chest tubes were typically removed on postoperative day 7 .

Table 1. Clinical characteristics of patients

\begin{tabular}{|c|c|c|c|c|c|c|c|c|}
\hline Case & Sex & Age (yr) & BMI $\left(\mathrm{kg} / \mathrm{m}^{2}\right)$ & Comorbidities & ASA grade & Neoadjuvant CCRT & Adjuvant CTx & Palliative RTx \\
\hline 1 & Male & 71 & 21.3 & No & 2 & No & No & No \\
\hline 2 & Male & 53 & 20.3 & Gastric ulcer & 1 & No & No & No \\
\hline 3 & Male & 70 & 21.3 & HTN, Pul. tbc, BPH & 2 & No & No & No \\
\hline 4 & Male & 71 & 23.2 & Alcoholic LC & 2 & No & Done & No \\
\hline 5 & Male & 54 & 17.5 & Pul. tbc, fatty liver & 4 & No & Done & Done \\
\hline 6 & Male & 65 & 18.9 & Pul. tbc & 2 & No & Done & Done \\
\hline 7 & Male & 46 & 23.5 & DM, HTN & 1 & No & Done & No \\
\hline 8 & Male & 64 & 23.8 & No & 2 & No & No & No \\
\hline 9 & Male & 65 & 24.6 & HTN & 1 & No & No & No \\
\hline 10 & Female & 42 & 23.7 & Schiz & 2 & No & No & No \\
\hline 11 & Male & 73 & 19.6 & HTN & 2 & No & No & No \\
\hline 12 & Male & 70 & 22.7 & No & 3 & No & Done & Done \\
\hline 13 & Male & 68 & 17.8 & HTN, COPD & 2 & No & Done & No \\
\hline 14 & Male & 60 & 25.0 & HTN & 2 & No & No & No \\
\hline Mean & & 62.3 & 21.7 & & 2 & & & \\
\hline
\end{tabular}

BMI, body mass index; ASA, American Society of Anesthesiologists; CCRT, concurrent chemoradiation therapy; CTx, chemotherapy; RTx, radiation therapy; HTN, hypertension; Pul. tbc, pulmonary tuberculosis; BPH, benign prostatic hyperplasia; LC, liver cirrhosis; DM, diabetes mellitus; Schiz, schizophrenia; COPD, chronic obstructive pulmonary disease. 


\section{RESULTS}

We performed LGPU in 14 consecutive patients. There was one 30-day postoperative mortality case. We only used the stomach for tube formation, and LGPU was performed for the esophageal cancer patients only, but not for patients with benign esophageal disease, such as esophageal stenosis.

\section{Clinical characteristics of patients}

Among 14 patients who had LGPU, only one patient was female, whereas the other 13 patients were male. Mean age was 62.3 years (range, $42-73$ years). Mean body mass index was $21.7 \mathrm{~kg} / \mathrm{m}^{2}$ (range, $17.5-25.0 \mathrm{~kg} / \mathrm{m}^{2}$ ). Mean American Society of Anesthesiologists (ASA) physical status classification score was 2 (range, 1-4).

Eleven patients had one or more comorbidities and three patients did not. The comorbidities of each patient are summarized in Table 1. No patient received neoadjuvant concurrent chemoradiation therapy (CRRT), six patients received adjuvant chemotherapy, and three patients received palliative radiation therapy (Table 1).

\section{Pathological characteristics of patients}

Esophageal cancer was located at the upper, middle, and lower esophagus for two, six, and six patients, respectively. All patients' biopsy results showed squamous cell carcinoma. For histologic type, three, eight, and three cases were well differentiated, moderately differentiated, and poorly differentiated, respectively. All resected specimens had tumor-free resection margin. According to pathological staging, two patients were in stage $\mathrm{I}_{\mathrm{A}}$, four patients in

Table 2. Pathological characteristics of patients

\begin{tabular}{ccclc}
\hline Case & Location of tumor & Pathology & Differentiation & Stage \\
\hline 1 & Middle & SCC & Moderate & $\|_{B}$ \\
2 & Middle & SCC & Well & $I_{A}$ \\
3 & Lower & SCC & Well & $\|_{B}$ \\
4 & Lower & SCC & Poor & IV \\
5 & Lower & SCC & Moderate & IV \\
6 & Middle & SCC & Moderate & $I_{\text {A }}$ \\
7 & Lower & SCC & Poor & IV \\
8 & Middle & SCC & Moderate & $I_{B}$ \\
9 & Upper & SCC & Moderate & $I_{B}$ \\
10 & Middle & SCC & Well & $I_{A}$ \\
11 & Upper & SCC & Moderate & $I_{B}$ \\
12 & Middle & SCC & Moderate & $\|_{B}$ \\
13 & Lower & SCC & Poor & $I_{C}$ \\
14 & Lower & SCC & Moderate & $I_{B}$ \\
\hline
\end{tabular}

SCC, squamous cell carcinoma.

Table 3. Surgical and early postoperative outcomes

\begin{tabular}{|c|c|c|c|c|c|c|c|c|c|c|c|c|c|c|}
\hline \multirow{2}{*}{ Case } & \multicolumn{3}{|c|}{ Operation time (min) } & \multirow{2}{*}{$\begin{array}{c}\text { Open } \\
\text { conversion }\end{array}$} & \multirow{2}{*}{$\begin{array}{l}\text { Blood loss } \\
(\mathrm{mL})\end{array}$} & \multirow{2}{*}{$\begin{array}{l}\text { Transfusion } \\
(\mathrm{mL})\end{array}$} & \multirow{2}{*}{$\begin{array}{l}\text { Postoperative } \\
\text { complications }\end{array}$} & \multirow{2}{*}{$\begin{array}{c}\text { Clavien- } \\
\text { Dindo } \\
\text { class }\end{array}$} & \multirow{2}{*}{$\begin{array}{c}\text { 30- } \\
\text { Day } \\
\text { mortality }\end{array}$} & \multirow{2}{*}{$\begin{array}{l}\text { ICU } \\
\text { stay } \\
\text { (day) }\end{array}$} & \multirow{2}{*}{$\begin{array}{c}\text { Hospital } \\
\text { stay } \\
\text { (day) }\end{array}$} & \multirow{2}{*}{$\begin{array}{l}\text { Chest } \\
\text { tube out } \\
\text { (day) }\end{array}$} & \multirow{2}{*}{$\begin{array}{c}\text { NG } \\
\text { tube out } \\
\text { (day) }\end{array}$} & \multirow{2}{*}{$\begin{array}{l}\text { Diet } \\
\text { start } \\
\text { (day) }\end{array}$} \\
\hline & Total & Chest & Abdomen & & & & & & & & & & & \\
\hline 1 & 375 & 250 & 125 & No & 1,000 & 1,680 & None & - & No & 2 & 17 & 7 & 3 & 3 \\
\hline 2 & 455 & 325 & 130 & No & 1,000 & 1,120 & None & - & No & 1 & 16 & 6 & 3 & 3 \\
\hline 3 & 335 & 215 & 120 & No & 2,000 & 1,600 & None & - & No & 1 & 17 & 6 & 3 & 3 \\
\hline 4 & 480 & 305 & 175 & No & 2,000 & 640 & None & - & No & 7 & 15 & 6 & 3 & 3 \\
\hline 5 & 455 & 305 & 150 & No & 1,800 & 1,440 & None & - & No & 1 & 23 & 7 & 4 & 4 \\
\hline 6 & 505 & 385 & 120 & No & 1,500 & 400 & None & - & No & 3 & 19 & 8 & 5 & 5 \\
\hline 7 & 430 & 250 & 180 & No & 300 & 0 & None & - & No & 1 & 14 & 7 & 4 & 4 \\
\hline 8 & 480 & 360 & 120 & No & 300 & 0 & $\begin{array}{l}\text { Anastomosis } \\
\text { site leakage }\end{array}$ & 1 & No & 2 & 26 & 7 & 4 & 4 \\
\hline 9 & 375 & 270 & 105 & No & 1,200 & 1,200 & $\begin{array}{l}\text { Pneumonia } \\
\text { SSI }\end{array}$ & $\begin{array}{l}\| \\
\text { I }\end{array}$ & No & 1 & 32 & 7 & 3 & 3 \\
\hline 10 & 395 & 305 & 90 & No & 500 & 0 & None & - & No & 2 & 22 & 7 & 3 & 3 \\
\hline 11 & 380 & 260 & 120 & No & 500 & 0 & $\begin{array}{l}\text { Aspiration } \\
\text { pneumonia } \\
\text { ARDS } \\
\text { Sepsis }\end{array}$ & $\begin{array}{l}\| \\
\mathrm{N}_{\mathrm{a}} \\
\mathrm{V}\end{array}$ & Yes & 16 & 16 & 16 & - & - \\
\hline 12 & 520 & 340 & 180 & No & 500 & 0 & SSI & I & No & 5 & 28 & 8 & 3 & 3 \\
\hline 13 & 387 & 257 & 130 & No & 500 & 640 & $\begin{array}{c}\text { Subcutaneous } \\
\text { emphysema }\end{array}$ & I & No & 3 & 21 & 10 & 3 & 3 \\
\hline 14 & 427 & 227 & 200 & No & 700 & 0 & $\begin{array}{l}\text { Pneumonia } \\
\text { SSI }\end{array}$ & $\begin{array}{l}11 \\
1\end{array}$ & No & 4 & 23 & 16 & 3 & 3 \\
\hline Mean & 428.5 & 289.6 & 138.9 & & 985.7 & 622.9 & & & & 3.5 & 20.6 & 8.4 & 3.4 & 3.4 \\
\hline
\end{tabular}

ICU, intensive care unit; NG, nasogastric; SSI, surgical site infection; ARDS, acute respiratory distress syndrome. 
stage $\mathrm{I}_{\mathrm{B}}$, three patients in stage $\mathrm{II}_{\mathrm{B}}$, one patient in stage $\mathrm{III}_{\mathrm{A}}$, one in stage III $_{C}$, and three patients in stage IV (Table 2).

\section{Surgical and early operative outcomes}

Mean total operation time (sum of abdominal and thoracic procedures) was 428.5 minutes (range, 335.0-520.0 minutes), and mean abdominal operation time was 138.9 minutes (range, 90-200 minutes). No case was converted to open surgery during LGPU. Mean blood loss was $985.7 \mathrm{~mL}$ (range, 300-2,000 mL), and mean transfused packed red blood cell volume was $622.9 \mathrm{~mL}$ (range, 0-1,680 $\mathrm{mL}$ ) on the first day postoperatively. We performed LGPU without any intraoperative complication, such as massive bleeding and so on. Five Clavien-Dindo classification I complications occurred. One patient had subcutaneous emphysema, which was treated by maintaining chest tubes. Three patients had surgical site infection (SSI) on thoracotomy sites, which were treated by antibiotics and frequent dressing. One patient had minor anastomosis site leakage detected on the esophagogram and recovered spontaneously without any special treatment. This patient showed anastomosis site stricture at 1 year postoperatively, and balloon dilatation was performed.

Three Clavien-Dindo classification II complications occurred. Two patients had pneumonia, which was treated by antibiotics and required no further treatment. However, one patient suffered from aspiration pneumonia, which developed into acute respiratory distress syndrome (ARDS; Clavien-Dindo classification $I_{\mathrm{a}}$ ). This patient died on postoperative day 16 due to sepsis (Clavien-Dindo classification V) at the ICU. The patient had a hypertensive history, which was well-managed by medication, and ASA score of 2 . We suspect that aspiration pneumonia occurred during extubation at the operating room. Patients spent an average of 3.5 days (range, 1-16 days) in the ICU. Mean hospital stay was 20.6 days (range, 1432 days). NG tube was removed on an average of 3.4 days (range, 3-5 days) postoperatively, and diet was started on an average of 3.4 days (range, 3-5 days) postoperatively. NG tube removal and diet start day were not included for the patient who died (Table 3).

\section{DISCUSSION}

The standard surgical treatment for esophageal cancer is esophagectomy, followed by esophageal reconstruction [8]. Anatomic characteristics such as absence of the serosal coating and connection with the periesophageal adventitia to the mediastinum, allows local invasion and distant metastasis to easily occur; consequently, en bloc surgery is not possible [9]. Hence, neoadjuvant chemotherapy is sometimes required preoperatively.

The stomach, jejunum, and colon are candidates for esophageal reconstruction. Denk [10] introduced esophageal reconstruction using the stomach from their animal experience, which they called transhiatal blunt total esophagectomy; it was approached through cervical and abdominal incisions. This procedure was reported in 1936 by Turner in living patients, but it had not been used since, due to its bleeding risk in the posterior mediastinum. Since then, Ong and Lee [11] reported successful treatment in three hypopharyngeal and cervical esophageal cancer patients. Thereafter, Le Quesne and Ranger [12] and Harrison and Thompson [13] reported modified procedures. This procedure, known as gastric transposition, is the method of pulling up the entire stomach and anastomosing the pulled-up stomach and pharynx. The stomach has been used for esophageal reconstruction owing to its several advantages, such as its relative ease in performance, reliable blood supply, adequate length, single anastomosis in the thorax, strong resistance to infection and acid exposure due to its thick muscular layer, and so on. We once performed colon interposition in our institution; however, our GI surgeons started performing gastric pull-up with tube formation since 2007.

The original Ivor-Lewis esophagectomy, first reported in 1946, combines an initial laparotomy and construction of a gastric tube, followed by a right thoracotomy to excise the tumor and a gastro-esophageal anastomosis [14]. It has become one of the main surgical procedures for the treatment of esophageal cancer.

Since laparoscopic cholecystectomy was first performed in 1989, laparoscopic surgery has been widely applied in patients with various gastrointestinal diseases. Laparoscopic surgery has several advantages over conventional open surgery, including lesser invasiveness, lesser pain, earlier recovery, and better cosmetic results. This minimally invasive surgical technique has been refined in conjunction with the development of advanced surgical instrumentation and has been applied to more complicated organ systems and disease processes at other institutions.

Our center developed and standardized operative techniques based on the multidisciplinary approach, wherein the abdominal laparoscopic component is performed by a GI surgeon, and the thoracic component is performed by a thoracic surgeon. Additionally, a different method of gastroesophageal anastomosis is used at each center. Nguyen et al. [15] also used a method similar to that used in our institution, such as placing the anvil via the thoracic cavity and purse-string suture. In 2009, Jeong and Park [16] reported the result of laparoscopic esophagojejunal anastomosis using an Orvil device. However, our center still uses Anvil placement in the esophagus and purse-string suture, which is a safe and secure method [17] for the following reasons: first, there are several restrictions for anastomosis using the Orvil device [15]; and second, additional purse-string suture is absolutely required if tightening of the anastomosis fails.

None of our patients had conversion to open surgery. We en- 
countered one case of minor anastomotic leakage, which was found by postoperative esophagogram, but it did not require any treatment. The patient was stable and was discharged with normal diet. Follow-up esophagography for this patient was performed at the outpatient department, revealing the absence of leakage. We had three SSI complications. SSI sites were not abdominal port insertion sites, but were open thoracotomy sites. Routine dressing was enough to treat this complication. Postoperative pneumonia was found in three patients who were then treated with antibiotics. However, we had one 30-day mortality case. This patient developed ARDS and died due to sepsis.

In our center, LGPU has been performed for abdominal procedures by a GI surgeon who has broad laparoscopic experience (over 200 cases of laparoscopic gastrectomy experience), and postoperative gastric stasis or passage disturbance has not been reported yet.

This study has several limitations. First, we had only 14 patients, which is relatively few compared to those included in other studies; hence, statistical analysis was unavailable for this study. Second, this study was retrospective in nature. However, the procedures were performed in a single center by a single GI surgeon, which could be viewed as a strength in this study. According to the study on the learning curve of minimally invasive esophagectomy by Tapias and Morse [18], 35 to 40 cases are considered as the learning curve. However, comparing the average abdominal procedure time, the operative time in Tapias and Morse [18] was 163.5 minutes, whereas, in this study, it was 138.9 minutes. The extensive experience of our GI surgeon is considered to be the reason our study can sufficiently address the limitation of a small number of cases.

If a GI surgeon has extensive experience in performing laparoscopic procedures, LGPU in thoracic esophagectomy will be performed safely and feasibly to treat patients with intrathoracic esophageal cancer. Moreover, we think the surgical outcomes would be better if the study involved more LGPU cases for esophageal cancer and if video-assisted thoracic surgery is added to the thoracic procedure.

\section{CONFLICT OF INTEREST}

No potential conflict of interest relevant to this article was reported.

\section{REFERENCES}

1. Thomas P, Fuentes P, Giudicelli R, Reboud E. Colon interposition for esophageal replacement: current indications and long-term function. Ann Thorac Surg 1997;64:757-64.

2. Spitz L. Esophageal replacement: overcoming the need. J Pediatr Surg 2014;49:849-52.
3. Bax KM. Jejunum for bridging long-gap esophageal atresia. Semin Pediatr Surg 2009;18:34-9.

4. Law S, Fok M, Chu KM, Wong J. Thoracoscopic esophagectomy for esophageal cancer. Surgery 1997;122:8-14.

5. Vierra M. Minimally invasive surgery. Annu Rev Med 1995;46: $147-58$.

6. Li B, Chen H, Xiang J, Zhang Y, Li C, Hu H, et al. Pattern of lymphatic spread in thoracic esophageal squamous cell carcinoma: a single-institution experience. J Thorac Cardiovasc Surg 2012; 144:778-85.

7. Yagmurlu A, Barnhart DC, Vernon A, Georgeson KE, Harmon $\mathrm{CM}$. Comparison of the incidence of complications in open and laparoscopic pyloromyotomy: a concurrent single institution series. J Pediatr Surg 2004;39:292-6.

8. Ellis FH Jr. Standard resection for cancer of the esophagus and cardia. Surg Oncol Clin N Am 1999;8:279-94.

9. Weijs TJ, Goense L, van Rossum PS, Meijer GJ, van Lier AL, Wessels FJ, et al. The peri-esophageal connective tissue layers and related compartments: visualization by histology and magnetic resonance imaging. J Anat 2017;230:262-71.

10. Denk W. Zur Radikaloperation des Osophaguskarfzentralbl. Chirurg 1913;40:1065-8.

11. Ong GB, Lee TC. Pharyngogastric anastomosis after oesophago-pharyngectomy for carcinoma of the hypopharynx and cervical oesophagus. Br J Surg 1960;48:193-200.

12. Le Quesne LP, Ranger D. Pharyngolaryngectomy, with immediate pharyngogastric anastomosis. Br J Surg 1966;53:105-9.

13. Harrison DF, Thompson AE. Pharyngolaryngoesophagectomy with pharyngogastric anastomosis for cancer of the hypopharynx: review of 101 operations. Head Neck Surg 1986;8:418-28.

14. Lewis I. The surgical treatment of carcinoma of the oesophagus; with special reference to a new operation for growths of the middle third. Br J Surg 1946;34:18-31.

15. Nguyen NT, Follette DM, Lemoine PH, Roberts PF, Goodnight JE Jr. Minimally invasive Ivor Lewis esophagectomy. Ann Thorac Surg 2001;72:593-6.

16. Jeong O, Park YK. Intracorporeal circular stapling esophagojejunostomy using the transorally inserted anvil (OrVil) after laparoscopic total gastrectomy. Surg Endosc 2009;23:2624-30.

17. Li H, Hu B, You B, Miao JB, Fu YL, Chen QR. Combined laparoscopic and thoracoscopic Ivor Lewis esophagectomy for esophageal cancer: initial experience from China. Chin Med J (Engl) 2012;125:1376-80.

18. Tapias LF, Morse CR. Minimally invasive Ivor Lewis esophagectomy: description of a learning curve. J Am Coll Surg 2014;218: 1130-40. 